\title{
Path Planning Analysis of Decelerator Assembly Based on Virtual Technology
}

\author{
http://dx.doi.org/10.3991/ijoe.v11i4.4617 \\ Xiangyang JIN \\ Harbin University of Commerce, Harbin, China
}

\begin{abstract}
In examining the structural characteristics of the different parts of a decelerator, the general rules of $3 \mathrm{D}$ part modeling are summarized, a three-dimensional model of a gear bracket is established, and all marshaled parts are generated into the whole parts assembly by considering the assembly relations and hierarchical relations of the parts. Then the reducer assembly path is sketched out, the assembly sequence of parts is determined, and the final completion of assembly simulation is finished. Virtual assembly technology is conducive to the rapid introduction of technology in advanced design methods to improve product quality, reduce the cost of development, and shorten the development cycle, which lays the foundation of practical assembly and disassembly of parts.
\end{abstract}

Index Terms-Constrained matching, 3D modeling, virtual assembly, path planning.

\section{INTRODUCTION}

Virtual assembly refers to computer aided design technology that helps to make better design decisions related to assembly during the process of product design and to conduct assembling analysis on the computer data model in a virtual environment. It combines virtual reality technology, advanced visualization technology, simulation techniques, decision-making theory, assembling and manufacturing techniques, etc. [1].

The key to inspection in traditional design, which decides whether a design is correct or not, is making use of a sample. When there is an unsuccessful assembly between one component and another, they must be returned to assembly for redesigning, reproducing of a sample and retesting with real assembly [2]. However, similar to computational realization in a real process, virtual assembly technology conducts modeling and simulation through computers, being able to inspect coordination and ability to be assembled among the components, to ensure the correctness of the design, to reduce the burdens of design rework, to shorten the development time and to save the manufacturing cost of prototypes [3]. Virtual assembly design systems are able to pre-assemble designed 3D models, avoiding the application of a physical model. More importantly, it gives full consideration to the assembly links of products and the influences of various related factors through different technological means like analysis, evaluation, programming and simulation. It improves the assembly structure of a product under the condition of satisfying performances and the functions of a product, enhances ability to assemble a product, reduces assembly costs and total costs of product, and shortens the time required for design.
A snow cleaning vehicle decelerator has a complex structure, high precision parts, and a difficult assembly process, so a reasonable assembly sequence is efficient. Based on the demand for virtual assembly processes, this paper puts forward a kind of simulation method and analyzes the relationship between the assemblies of complex parts. The relational model of assembly parts is established by layer assembly: the sub assembly layer, part layer, feature layer, and component geometry layer. The model is reproduced by three dimensional virtual image technology. Test results show that the removing and cross assembly processes of complex parts are reproduced well based on virtual 3D images of the complex parts assembly process.

Virtual technology is an important part in the enterprise informatization as the process data greatly generates support for the company's production activity. Virtual assembly is a hot spot in the research of Virtual Reality, which has vital significance to design confirmation and solution for product assembly order and provides further support for complicated product assembly design. It operates dynamic simulation of the product assembly process by manipulating part models directly in the virtual environment and checks the feasibility of assembly and validity of the assembly path. Based on the wide use of three dimensional CAD and the advantage of virtual assembly technology, this paper introduces a computer aided assembly process plan system based on virtual technology from the angle of the entire process of three dimensional product data in machinery assembly design and then investigates the system structure, realization method and key techniques.

\section{THE 3D MODELING OF SINGLE GEAR STAND}

The new type of decelerator with an adjustable gap is mainly composed of a gear shaft, gear, gear stand, bearings, end caps and other components. Each component has unique structure characteristics, and different modeling methods are required.

Although structural styles of mechanical products differ in thousands of ways (as do applications and working principles), there are still some rules can be found in 3D solid modeling on a computer [4]. Generally speaking, for rotating components like an end cap, gasket, retainer ring and others, a sketch of the sectional view can be drawn first to generate the main body through contour rotation, to be modified with chamfering tools and hole tools, and finally to set the required number by means of mirror images and arrays [5-8]. Thus, an integrated 3D solid model is achieved. The general process and main methods 
of decelerator 3D modeling are illustrated with the example of a gear stand.

\section{A. The Main Body of Gear Stand}

First, generate the main body of a gear stand with a "Rotation" tool in the "Insert" menu [9]. In this paper, RIGHT datum level was selected as the sketch plane. Then, draw the 2D section shown in Fig. 1 and Fig. 2. The solid line shows the cross-section shape and the dashed line at the bottom represents the spin axis.

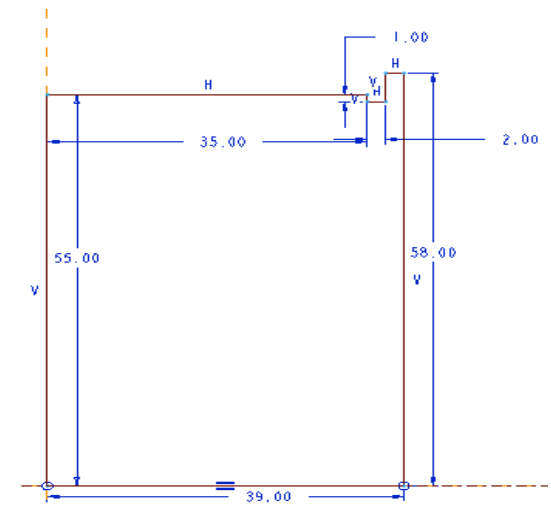

Figure 1. Sketch of rotating section

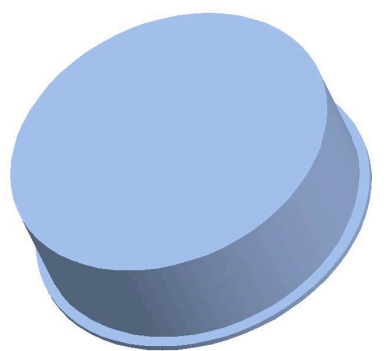

Figure 2. Rotation-generated entity

\section{B. Truncate Slots and Holes}

Use the "Stretching" tool and click the "Remove Material" button. Select the TOP datum level, which is the end face of the gear stand, to draw an appropriate section sketch. Stretch according to the required length. The model that is shown in Fig. 3 was obtained after four times of stretching in succession.

\section{Drill Holes with Drilling Tool}

Two kinds of holes can be placed in Pro/E, namely a straight hole and a gauge hole, which can be formed through selecting the location, dimension and depth of the hole. Straight holes can be divided into two categories, a simple straight hole and a sketched hole. A simple straight hole is commonly used for drawing through-holes, while a sketched hole is quite suitable for drawing blind holes with conicity at the bottom. What should be noticed is that the spin axis should be vertically placed in sketching and the top of the sketched section must be a horizontal straight line standing for the plane of holes that can be placed. A gauge hole, i.e., a threaded hole, can be selected in accordance with the related standard of thread. Because the holes on both sides are symmetric, drill holes on one side and then on the other side can be obtained conveniently through the copy feature. The 3D model formed after drilling three pairs of holes is in Fig. 4.

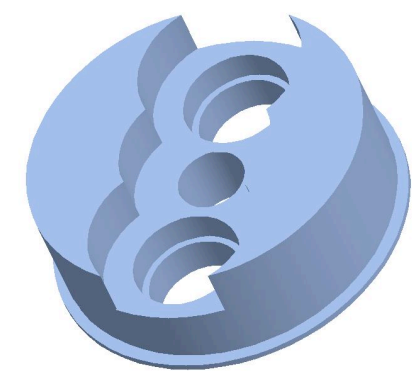

Figure 3. Model after stretching and removal of material

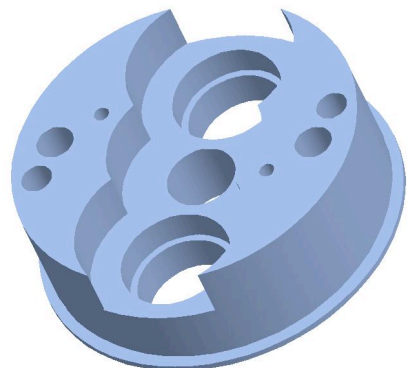

Figure 4. Gear stand model after slotting

\section{Establishment and Modification of Features of Boss}

Roll the model over and then stretch and image the boss at the datum level of the end face of the blank, which is shown in Fig 5-a. Drill holes twice at the datum level of the top plane of the boss and make a copy, which is shown in Fig 5-b. The smaller ones are threaded holes, while the bigger ones are bore-holes. The drawing of the gear stand model is completed.

3D solid models of all components can be built with similar approaches. 3D models of other major components are shown in Fig. 6.

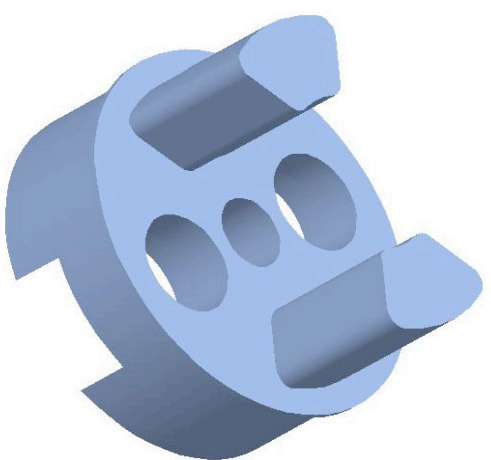

a) Model with stretched boss

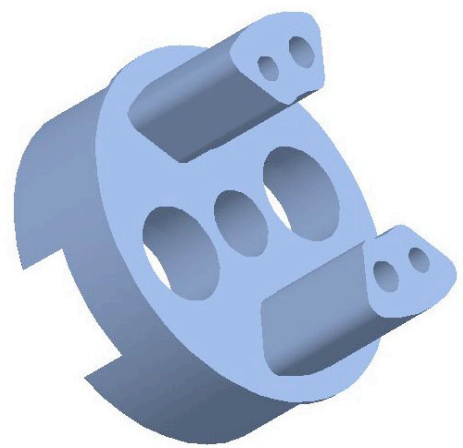

b) Completed model

Figure 5. Finish the drawing of 3D solid model of gear stand 


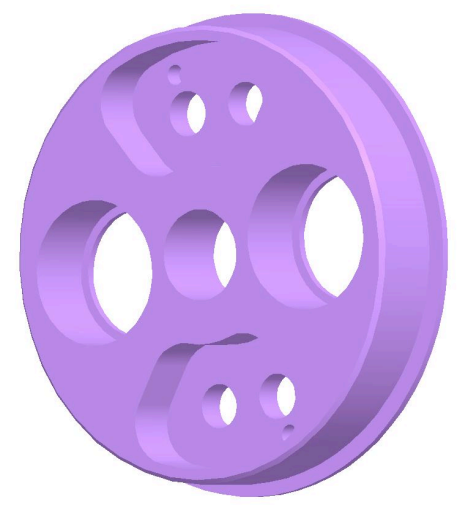

a) Lateral plate of stand

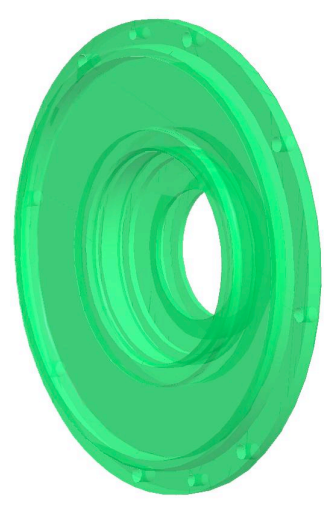

b) End cap

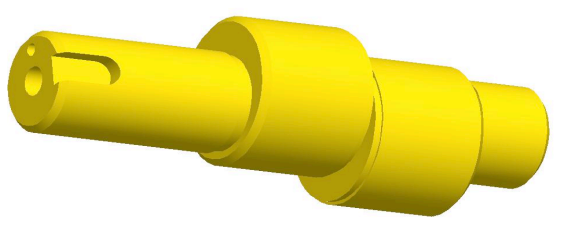

c) Double eccentric shaft

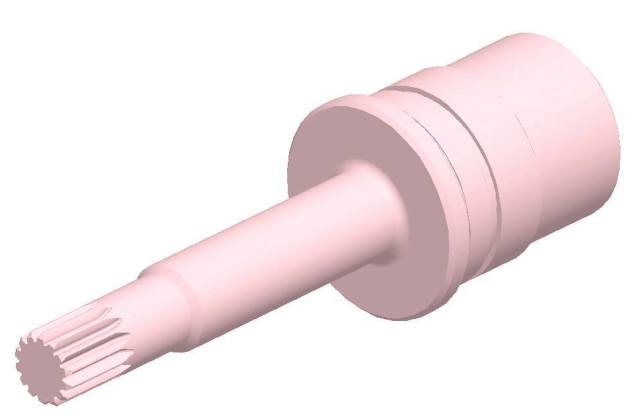

d) Gear shaft

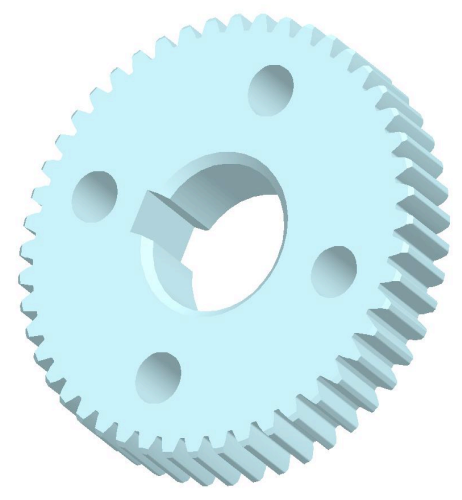

e) Planetary gear

Figure 6. 3D solid models of major components of decelerator

\section{VIRTUAL ASSEMBLY OF DECELERATOR}

\section{A. Virtual Assembly Approaches}

Decelerator assembly starts from models of the components, which can be transferred or created at any time. The most important step in components assembly is to restrain components properly. There are several ways of component assembly provided by Pro/E, including matching, aligning, inserting, coordinate system, tangency, point on line, point on curved surface, side on curved surface, etc. Afterward set the connection between the components, which means to establish the constraint relations between the components in line with matching the conditions and determining their positions.

Based on the theory of single data dependency, a Pro/E assembly system assembles geometric solids of components instead of making duplications during the whole virtual assembly. Therefore, no matter how or where components are edited, assembly parts can be updated automatically, reflecting the latest changes of the components.

\section{B. Assembly Path Planning}

There are two kinds of assembly path planning in terms of realization, namely automatic calculation planning and manual planning. Automatic calculation planning is achieved automatically through the assembling relation between matching surfaces of components, which is still not quite well-developed. Manual planning is realized through human-computer interaction, which gives full play to people's subjective initiative and guarantees the correctness of planning. In this paper, the manual assembly process of product is simulated and the disassembly process of a new decelerator with an adjustable gap is studied in a virtual environment, determining the movement direction and order of each component during assembly and disassembly.

First, install tin bronze gaskets on the two eccentric shafts to separate the two thickened external gears and reduce friction. Afterward, install the thickened external gears (with a bearing inside the hole) and then install the gear stand and lateral plate of the stand through bolting (with a locked gasket) to make a fixed assembly by drilling and slotting the pin holes and driving in male pins. Install four small bearings supporting the double eccentric shaft, and fix shafts on one end of the lateral side of stand through the retainer ring of the eccentric shaft, locked 
gasket and threaded bolt. Install the shells of the two parts (ensuring correct meshing between the inside and outside thickened gears) and shafts. Then, assemble the planetary gear, components of the gear shaft and components of the planetary gear in correct meshing. Drill the pin holes and slot locating pins for components of the planetary gear, and then install a sealed end cover (with an O-ring).

In the assembly of the gear shaft components, install the bearing and elastic retainer ring for the shaft on the gear shaft, which then should be put into the end cover hole with a sealed ring. At last, fix the elastic retainer ring for the hole.

The disassembling process of the decelerator is the inverse process of the assembly, following the reverse order and path. In the disassembling process of this decelerator, the components make a rectilinear movement basically in the axial direction of the decelerator.

\section{The Virtual Assembly Process of Decelerator}

An entire assembly model consists of multiple subassemblies and components. Appropriate sub-assemblies can largely reduce the work of assembly. For example, a bolt or a screw usually appears with a matched spring washer at the same time. It is very inconvenient to assemble one by one if two components must be used each time. Therefore, they should be combined as a sub-assembly. There are two eccentric shafts with symmetrical structures, which can be combined with related a bearing, retainer ring and bolt as a sub-assembly. The gear stand, lateral plate of the stand and related screws and pins can be assembled together as a component, defined as a subassembly.

General steps of building up an assembly are as follows: First, set up a component selectively and start the assembling mode. Then import the first positioning element, to which the system gives a "fixed" position automatically and makes the degree of freedom zero, becoming the standard of placing other components in the assembly. Afterward other components or sub-assemblies can be imported or built up successively. Position each in accordance with various constraint methods provided by the Pro/E until assembly is completed. The 3D solid model of the assembled decelerator is shown in Fig. 7.

To show the internal structure clearly, the outermost components: the shell, end cap and sealed end cap are processed with verification. And different colors are used in each component to conveniently identify and distinguish each one.

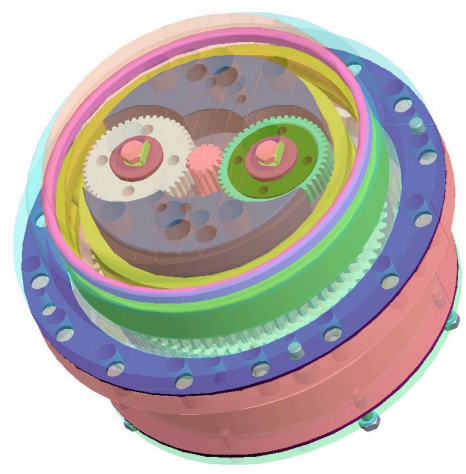

Figure 7. 3D solid models of the virtual assembly of decelerator

\section{CONCLUSION}

To build a flexible assembly model, import single components into the system and organize the components into groups to form a whole assembly; add different kinds of assembling relations between the components and in relation to the assembly level.

Program the assembly path of the decelerator, determine the order of the component assembly, achieve simulation of the assembly, and lay the foundation for the actual assembly and disassembly of the components.

Introduce various constraint methods of virtual assembly provided by Pro/E software and identify their application scope and approaches. Propose the general rule of virtual assembly in the environment of Pro/E and complete assembly of the 3D solid model of a decelerator.

\section{REFERENCES}

[1] Changpei Wang. "Research on virtual demonstration of historical and cultural sites based on 3D laser scanning technology," International Journal of Digital Content Technology and its Applications, vol. 6, no. 2, pp. 137-144, February 2012.

[2] Yamato, Yoji. "Automatic verification technology of software patches for user virtual environments on IaaS cloud," Journal of Cloud Computing, vol.4, no.1, pp. 1-14, 2015. http://dx.doi.org/10.1186/s13677-015-0028-6

[3] Shen Wenchen, Hu Yuhui, and Yu Huilong. "Shifting process control of AMT based on virtual clutch technology for hybrid electric vehicle," Journal of Mechanical Engineering, vol. 50, no. 18, pp.108-117, September 2014. http://dx.doi.org/10.3901/ JME.2014.18.108

[4] Lan Yuqing, Xu Hao. "Research on technology of desktop virtualization based on SPICE protocol and its improvement solutions," Frontiers of Computer Science, vol. 8, no. 6, pp. 885-892, November 2014. http://dx.doi.org/10.1007/s11704-014-3410-5

[5] Park Hong-Soek, Le Ngoc-Tran. "Modeling and controlling the mobile harbour crane system with virtual prototyping technology," International Journal of Control, Automation and Systems, vol.10, no.6, pp.1204-1214, December 2012. http://dx.doi.org/10.1007/ s12555-012-0615-y

[6] Matsui Kazuki, Horio Kenichi, and Sato Yuichi. "Virtual desktop display acceleration technology: RVEC," Fujitsu Scientific and Technical Journal, vol.48, no.4, pp.469-475, October 2012.

[7] Xue Peiyun, Zhang Xueying, Bai Jing. "Research of disk migration technology for virtual machine in cloud computing," Journal of Computers (Taiwan), vol.24, no.3, pp. 3-10, October 2013.

[8] Jin Xiangyang, Sun Zhihui, and Wang Heteng. "Application of improved support vector machine regression analysis for mediumand long-term vibration trend prediction," Journal of Vibroengineering, vol. 15, no. 2, pp. 942-950, 2013.

[9] Villa D., Gazzola J., and Dal Fabbro I. M. "Moir supported stress distribution study on gears." INMATEH - Agricultural Engineering, vol. 44, no. 3, pp. 157-164, 2014.

\section{AUTHOR}

Xiangyang Jin, born in 1976, received a Ph.D. in Mechanical Engineering from Harbin Institute of Technology, China in 2012. Currently, he is a researcher at Harbin University of Commerce, Heilongjiang Province, China. His research interests include mechanism design theory, product innovation design and vibration signal analysis (email: jinxiangyang@126.com).

This work was supported by Applied Technology Research and development Project of Heilongjiang province (GC13C206). Submitted, April 9, 2015. Published as resubmitted by the authors on June 25, 2015. 